\title{
Modulation of taxonomic (versus thematic) similarity judgments and product choices by inducing
}

\section{local and global processing.}

Duncan Guest (uncan.guest@ntu.ac.uk) ${ }^{\text {abc }}$

Michael Gibbert $^{\mathrm{c}}$

Zachary Estes $^{b}$

David Mazursky de

Michael Lam ${ }^{\mathrm{a}}$

a Division of Psychology, Nottingham Trent University, UK

${ }^{\mathrm{b}}$ Department of Marketing, Bocconi University, Italy.

${ }^{\mathrm{c}}$ Institute of Marketing and Communication, University of Lugano, Switzerland,

${ }^{\mathrm{d}}$ The Jerusalem School Of Business Administration, The Hebrew University Of Jerusalem, Jerusalem, Israel,

e The Center for Academic Studies, Or Yehuda, Israel.

Contact details

Duncan Guest (corresponding author) - Psychology Division, Nottingham Trent University, Burton Street, Nottingham, UK, NG1 4BU. Tel: +44(0)115 84 82701. duncan.guest@ntu.ac.uk

Zachary Estes, Associate Professor, Department Of Marketing, Bocconi University, Via Roentgen, 1, Milano 20136, Italy estes@ unibocconi.it

Michael Gibbert, Professor Of Marketing, Institute Of Marketing and Communication, University Of Lugano, Via Giuseppe Buffi 13. Ch-6904 Lugano, Switzerland. michael.gibbert@usi.ch

David Mazursky, Kmart Professor Of Marketing, The Jerusalem School Of Business Administration, The Hebrew University Of Jerusalem, Jerusalem, Israel msmazur@ huji.ac.il 


\begin{abstract}
Perceived similarity is influenced by both taxonomic and thematic relations. Assessing taxonomic relations requires comparing individual features of objects whereas assessing thematic relations requires exploring how objects functionally interact. These processes appear to relate to different thinking styles: abstract thinking and a global focus may be required to explore functional interactions whereas attention to detail and a local focus may be required to compare specific features. In four experiments we explored this idea by assessing whether a preference for taxonomic or thematic relations could be created by inducing a local or global perceptual processing style. Experiments 1-3 primed processing style via a perceptual task and used a choice task to examine preference for taxonomic (versus thematic) relations. Experiment 4 induced processing style and examined the effect on similarity ratings for pairs of taxonomic and thematically related items. In all cases processing style influenced preference for taxonomic/thematic relations.
\end{abstract}

Keywords: Categorization; similarity; thematic relations; processing style; consumer behaviour. 


\section{Modulation of taxonomic (versus thematic) similarity judgments and product choices by}

\section{inducing local and global processing.}

When determining similarity between concepts, previous research has highlighted a distinction between taxonomic similarity and thematic similarity. Often similarity is described as the degree to which two things share or differ on a set of features (e.g., Gentner \& Markman, 1997; Tversky, 1977). For example a dog and cat both have tails and fur and they both are warm blooded and bear live offspring but differ in other aspects such as their ability to climb and number of teeth. These taxonomic features tell us that both can be placed into the "mammal" category but that cats and dogs are not the same type of mammal. Taxonomic relations therefore serve to group and differentiate objects in the world (Markman \& Wisniewski, 1997).

Although important, taxonomic relations do not determine similarity entirely; thematic relations also have a large influence on similarity. Items are related thematically if they functionally interact in the same scenario (Estes, Golonka, \& Jones, 2011), that is, if they perform complementary roles in a given situation. For example, a helmet has a number of functions, but when used in combination with a motorbike a helmet protects the head in an accident and keeps the face shielded from wind and rain. Both of these are important, but only in the context of riding a motorcycle at speed. Thematic relations are therefore about complementary rather than similarity between features. Thematically related items can be similar in terms of features, but tend not to be because for two concepts to perform different and complementary roles, they ordinarily must have different features. Nevertheless, thematic relations do influence perceptions of similarity (Estes, 2003; Golonka \& Estes, 2009; Simmons \& Estes, 2008). For example, Wisniewski and Bassok (1999) asked participants to rate how similar pairs of objects were. The pairs were taxonomically related (e.g., ship \& canoe) and/or thematically related (e.g., ship \& sailor). Similarity ratings were highest for object pairs that were both taxonomically and thematically similar (e.g., ship \& tugboat) compared to object pairs that were only taxonomically or thematically related, indicating that the thematic relations contributed to overall similarity judgments. Both taxonomic relations and thematic relations therefore contribute to assessments of similarity.

Critically, thematic and taxonomic relations have unique and contrasting characteristics (Estes et al., 2011). Thematic relations are external in that they occur between multiple objects, concepts, people, or 
events. They are also characterized by functional integration, whereby the things must perform complementary roles in that relation. In contrast, taxonomic relations are characterized by internality in that they are based on the features of the objects themselves. Due to the different processes involved, neuropsychological evidence indicates that taxonomic and thematic relations are processed in anatomically distinct cortical networks. For example, the left temporo-parietal cortex is more strongly activated during thematic processing than during taxonomic processing (Kalénine et al., 2009). Furthermore, localized damage to the left anterior temporal lobe is associated with taxonomic impairment, whereas damage to the left temporo-parietal cortex is associated with thematic impairment (Mirman \& Graziano, 2012a; Schwartz et al., 2011). Taxonomic and thematic processing also elicit distinct patterns of neural oscillation (Maguire, Brier, \& Ferree, 2010). Overall then, it appears that there is considerable research indicating that thinking about taxonomic and thematic relations relies on different processes that are neurologically distinct (see Estes et al., 2011 for a recent review).

Interestingly, it appears that individuals have a bias for taxonomic or thematic relations, and that this bias is stable across tasks. In a screening task Gentner and Brem (1999) presented a set of triads in which there is a base item (e.g., dog) and participants choose whether a taxonomically related item (e.g., cat) or a thematically related item (e.g., bone) is most similar. Although the majority of participants showed no preference, $48 \%$ consistently chose taxonomically related items whereas $11 \%$ consistently chose thematically related times. Similarly, across three experiments Simmons and Estes (2008) found consistent preferences for taxonomic or thematic relations. In their experiments, participants completed triad tasks in which typically around a third consistently chose thematic options whereas a third consistently chose taxonomic options. This choice consistency was demonstrated across triad tasks asking people to choose the most "similar" item, most "different" item and the item most "like" the base item. Similar consistency was observed when participants made similarity ratings between pairs of concepts.

Surprisingly this individual preference does not appear to be due to the demand characteristics of making explicit judgements. Mirman and Graziano (2012b) got participants to complete a looking task in which eye movements were recorded before a triad task. In the looking task, participants were shown four images, two of which were either related taxonomically or thematically, and two of which were unrelated to 
the other pictures. After the pictures had been presented for a $1300 \mathrm{~ms}$ a word was played through speakers and participants either clicked on the corresponding picture (active condition) or did nothing (passive condition). Analysis showed that, after word onset the picture corresponding to the word was looked at most, but also that the related picture was looked at more than the unrelated pictures. The extent to which taxonomically related pictures captured attention relative to the thematically related pictures was calculated as an implicit measure of taxonomic preference. This was shown to significantly predict the degree to which participants made taxonomic choices in the subsequent triad task.

Taken together, these studies suggest that the way in which people think about the relations between concepts may be relatively fixed. The question that we ask in the present study is whether preference for taxonomic/thematic relations may be malleable and whether it can be altered by a seemingly unrelated task. Surprisingly, little work has examined the antecedents of thematic and taxonomic judgements despite the different processes they appear to entail. Assessing thematic relations requires integration, that is, examining how things can relate and interact with each other. This seemingly requires a degree of abstract thinking, as the interactions will typically not be based upon perceptual similarity (e.g. features). Thus it is about considering an object as a whole in terms of its potential uses and functions and considering how these relate to other objects. This type of abstraction is aligned with what is often called global processing (e.g., Burgoon, Henderson \& Markman, 2013, Dijkstra et al, 2012, Huntsinger, Clore \& Bar_Anan, 2012). In contrast, assessing taxonomic similarity requires focusing on the specific feature attributes of objects and comparing these. This means breaking down items into their constituent parts in order to compare them. This style of information processing is more concrete (i.e., less abstract) in nature as it relies upon specific detail of numerous features. The focus on parts rather than the whole means that this type of processing is often referred to as local processing (e.g., Burgoon, Henderson \& Markman, 2013, Dijkstra et al, 2012, Huntsinger, Clore \& Bar_Anan, 2012). It seems then that thinking thematically or taxonomically might well be aligned with a global and local processing style respectively. Processing conceptual information globally or locally has also been linked to processing the perceptual world in a global (e.g.. attention to the whole display) or local (e.g., attention to parts of the display) manner (Derryberry \& Tucker, 1994; Forster \& Dannenberg, 2010). A wealth of research has therefore looked at manipulating attentional focus in order to 
investigate its effect on social or cognitive judgements (for a review see Burgoon, Henderson \& Markman, 2013). This raises the possibility that inducing a local or global perceptual focus could influence preference for taxonomic or thematic relations. This paper aims to examine whether this is the case.

Further support for the notion that global and local processing may influence the extent of taxonomic and thematic thinking comes from the relationship between a difference and similarity focus. Determining similarity involves assessing both commonalities and differences between concepts (Tversky, 1977) and these appear to be differentially weighted when thinking about thematic and taxonomic relations.

Taxonomic thinking involves comparison of features whereas thematic thinking requires integrating items or concepts. Gentner and Gunn (2001) induced taxonomic or thematic thinking by asking participants to compare (taxonomic thinking involves comparison of features) or integrate (thematic thinking requires integrating concepts) a pair of concepts and then list differences between the concepts. More differences were listed if the concepts had previously been compared, suggesting an association between thinking taxonomically and detecting differences. In contrast, thematic processing appears to involve a greater focus on commonalities than on differences. Golonka and Estes (2009) asked participants to rate either how different or how similar concepts were. Similarity judgments were influenced much more by thematic relations than were difference judgments, indicating that thematic processing was associated with identifying commonalities (which have a greater influence on similarity judgments) rather than differences. Conceptually, thematically related items tend to differ in their features (as a functional interaction often requires different features) and can only be understood by focusing on commonalities, with only a single commonality required to make items thematically similar. In contrast, taxonomic processing involves assessing both the commonalities and the differences between items and weighing these up in order to deduce similarity. Therefore taxonomic processing is more reliant on processing differences than thematic processing. Importantly, thinking about similarities or differences has been used as another way of manipulating abstract or concrete thinking alongside inducing a global or local processing (Fujita \& Roberts, 2010; Mullen, Pizzuto, \& Foels, 2002; for a review see Burgoon, Henderson \& Markman, 2013) ${ }^{1}$. We therefore also examine whether inducing a local/global focus leads to a difference in focus on similarities or differences, although this was not the central research question. 
In four experiments we examine whether manipulating perceptual processing style influences the degree of taxonomic thinking. We induced either a local attentional focus (focusing on small details or parts of objects) or a global attentional focus (attending to the "whole" rather than individual parts). Such perceptual processing is linked with conceptual processing and has previously been used widely to induce more abstract (global) or more concrete (local) thinking (Burgoon, Henderson \& Markman, 2013, Dijkstra et al, 2012, McCrea, Wieber \& Myers, 2012). We predicted that local and global processing would therefore induce taxonomic and thematic thinking, respectively. A set of experiments tested this general hypothesis by examining whether processing style influenced similarity judgments. In Experiment 1 participants were given a change blindness task in which they searched for a change between two successive images or were asked to produce a caption describing what was going on in the picture or were given no task (control condition). The change blindness task was intended to induce local processing by making participants systematically focus on small parts of the picture whereas the caption task was intended to produce global processing by making participants focus on the entire image. Participants' preference for taxonomic relations was then measured using a choice task in which they chose which two items were most similar. Participants were also asked to report differences and commonalities between other pairs of concepts in order to examine the relation between global/local processing and a similarity/difference focus as such a focus might support thematic/taxonomic thinking. Experiment 2 examined whether inducing a difference focus would still influence preference for taxonomic relations in a choice task even when the task did not explicitly ask about similarity relations. Experiment 3 examined whether preference for taxonomic relations could still be influenced by using a different task to induce local processing. Experiment 4 used a within subject design to establish whether similarity judgements could be influenced within the same experimental session.

\section{Experiment 1}

In Experiment 1 participants' attentional focus was either primed or not and then participants completed a triad choice task to measure preference for taxonomic (versus thematic) relations. After the 
choice task participants listed differences or similarities between pairs of objects in order to explore whether priming also influenced a focus on differences and similarities.

\section{Method}

Participants. The control, global processing and local processing groups comprised 97, 47 and 47 participants. Participants were from a University in Milan, Italy. All participants (here and in subsequent experiments) were studying marketing in English speaking classes. Nevertheless all materials were presented in both English and Italian.

Materials Design and Procedure. The experiment (and Experiments 2 and 3) took place in a lecture theatre at the start of a class, thus all groups were in different classes. All participants in each group completed the task at the same time. The difference focus was induced by a PowerPoint presentation shown on a large screen at the front of the lecture theatre. The presentation showed participants a series of eight change blindness trials. On each trial, two pictures (photos) were presented alternately with an interleaving grey display for 30 seconds. The pictures were identical except for a small detail. Pictures were taken from a Change Blindness program created by the Cognitive Science department at Indiana University which is freely available to download and use at http://cognitrn.psych.indiana.edu/CogsciSoftware/ChangeBlindness/\#examples. Participants searched for this changing detail and wrote this down within the 30s window the stimulus was shown for. Two practice trials preceded eight experimental trials. In the global processing group, the same eight pictures were displayed (the versions with a single item missing were not included) and participants were asked to write a caption describing each picture. Each caption had to be written down in the 30s window the stimuli were presented for. The control group did not receive any processing style inducement.

All participants subsequently completed a choice task in which they were given a base word (e.g., dog) and then two responses, one of which was taxonomically related to the base word (e.g., cat), the other thematically related to the base word (e.g., bone). Nine triads were used (see Table 1) sampled from prior research (Simmons \& Estes, 2008). These stimuli were previously designed such that the taxonomic and thematic alternatives were matched for word frequency, strength of association with the base concept, and 
frequency of co-occurrence with the base concept. On completing the triad task, participants continued onto a questionnaire that asked them to list either differences $(\mathrm{N}=47,24$ from the local processing group and 23 from the global processing group) or similarities between items $(\mathrm{N}=47,23$ from the local processing group and 24 from the global processing group). Instructions were given on the questionnaire. Participants were given one minute for each pair to list differences, or similarities (commonalities) between four pairs of items. The pairs of items were chosen to range from very similar to very dissimilar: hamburger and sushi, watch and jewels, credit card and wallet, ipod and hotel.

\section{Results and Discussion}

Choice task. The percentage of taxonomic responses in Experiments 1 is shown in Figure 1. A one way between subjects ANOVA with condition (global, local, control) as a factor yielded a significant effect of condition, $F(2,188)=4.31, \mathrm{MSE}=.06, p=.015, \eta_{P}^{2}=.04$. Paired comparisons (Fisher's LSD) showed that there were significantly more taxonomic choices in the local condition than the global condition $(p$ $=.024)$, and significantly more taxonomic choices in the local condition than the control condition $(p$ $=.005)$. There was no difference between the control and global conditions $(p=.84)$. Inducing a local focus therefore made participants more likely to choose taxonomically related items in the triad task (relative to thematic relations). In other words, inducing a more concrete style of thinking in which local details are important appeared to support taxonomic processing which involves comparing the features of objects. Interestingly however, inducing global focus had no effect on taxonomic thinking. Before drawing further conclusions about why this might be the case it is important to establish whether this finding was due to any particular specifics of the task, either the choice task or the task used to induce a global/local focus.

Comparison task. The mean number of items listed for the difference task and commonality task for the local processing and global processing conditions of Experiment 1 is shown in Table 2. Overall more differences were generated than similarities. Of interest is whether the local processing inducement led to a greater number of differences being reported and a reduced number of similarities. This trend was apparent, and a between subjects ANOVA on the mean number of items listed with condition (local processing or global processing) and task (difference or similarity task) as factors yielded a significant main effect of task, 
$F(1,90)=13.40, M S E=8.43, p<.001 \eta_{P}^{2}=.13$, and a significant interaction between processing style and task, $F(1,90)=4.12, M S E=8.42, p=.045, \eta_{P}^{2}=.04$. Post hoc tests (Fisher's LSD $\left.{ }^{2}\right)$ indicated that the number of items listed differed between the difference and similarity listing tasks for the local processing group only $(p<.001)$. All other comparisons were non-significant (all $p>.13)$. Thus it seems that there is some evidence for a link between processing style and a focus on differences or similarities ${ }^{1}$. However, this was only clear when local processing was induced, which led to a greater focus on differences than similarities. As indicated above, there is evidence that taxonomic thinking is related to a focus on differences (Gentner and Gunn, 2001) and so such a difference focus could have supported the increased taxonomic thinking observed in the local condition.

Similarities and differences were also coded as being either taxonomic or thematic (see Table 2). As thematic relations typically are based on a single relation, the vast majority of items listed concerned taxonomic relations. The same ANOVA as performed on the overall number of items listed was performed separately for taxonomic items listed and thematic items listed. For taxonomic items listed the ANOVA yielded a main effect of the task, with participants providing more differences, $F(1,90)=20.67, M S E=$ $8.41, p<.001, \eta_{P}^{2}=.19$. The interaction between listing task and processing style was also marginally significant, $F(1,90)=3.92, M S E=8.41, p=.051, \eta_{P}^{2}=.04$. Post hoc tests (Fisher's LSD) indicated that the number of items listed differed between the difference and commonality listing tasks for the local processing group only $(p<.001)$. All other comparisons were non-significant (all $p>.15$ ). The ANOVA on thematic items listed yielded a main effect of the rating task only, with participants finding it easier to produce a thematic commonality than a thematic difference, $F(1,90)=9.12, M S E=.72, p=.002, \eta_{P}^{2}=.09$.

Breaking the items listed down into taxonomic and thematic categories shows a clear difference between the number of items of each type listed. However, due to the small number of thematic items reported the analysis of whether this differs per condition is of limited use. As outlined in the introduction, thematic similarity appears to be due to a single salient relation. Given its contribution to similarity (Gentner \& Gunn, 2001) clearly a single thematic relation may have much greater influence over similarity than the more numerous taxonomic relations. Moreover, the importance of the thematic relation listed will itself 
differ, potentially between conditions. Thus it is plausible that manipulating processing style will influence the relative importance allocated to thematic and taxonomic relations as well as the number of items listed.

As the effect of the processing style manipulation on taxonomic/thematic thinking was the primary aim of the paper, Experiments 2-4 concentrated on this analysis and examined the extent to which the effect observed in Experiment 1 was due to the task used.

\section{Experiment 2}

To establish the generality of the findings in Experiment 1, Experiment 2 used a similar method to induce processing style but differed in the type of choice task. In the choice task in Experiment 1 the stimuli were concepts and participants chose which item was most similar to the base item. Although useful, it is perhaps rare that such similarity choices are made between the pairings used in Experiment 1 (e.g., choosing whether a bone or cat is more similar to a dog). Experiment 2 therefore used a more real world version of the triad task in which participants were told to imagine "You have just bought product X (e.g., a TV), would you now prefer to buy product Y (e.g., a stereo), or product Z (e.g., a sofa)?” As in Experiment 1 there was always one taxonomically related item and one thematically related item. However, now all the items referred to products and the choice made was one of product purchase, not similarity. We hypothesised that similarity would underlie choices (e.g., Markman \& Loewenstein, 2010) such that making two items seem more similar will increase the chance that they are chosen together. This is based on the notion that purchasing is more likely to be structured and partially dependent on the relations between things rather than being unstructured (buying items that are unrelated). We therefore expected that increasing the similarity between products would make them more appealing. Of course, it is possible that similarity can be disadvantageous if the products perform competing roles, and this would reverse the effect of processing style observed in Experiment 1. However, stimuli were chosen such that they would not compete with each other and so we did not expect such a reversal.

In order that the task resembled a real life situation the items selected needed to be products that people might feasibly buy together. This meant taxonomically related pairs and thematically related pairs were made up of items that are used/consumed in a similar setting. Inevitably, a common usage situation 
creates the possibility of envisaging some form of functional interaction between the items, that is, a thematic relation. Thus, it is possible that participants would see some kind of thematic relation for the taxonomically related items. Overall however, the taxonomic items were characterised by high taxonomic similarity (a lot of similar features) and low thematic similarity whereas the thematic items were characterised by high thematic similarity and low taxonomic similarity.

\section{Method}

Participants. The control, global processing group and the local processing group comprised 37, 32, and 30 participants respectively, from a University in Lugano, Switzerland.

Materials and Procedure. The procedure was the same as that in Experiment 1 except that in the triad task participants were told to imagine that they had just bought the base word, and were asked which alternative they would now prefer to buy. Twelve triads were used (see Table 3).

\section{Results and Discussion}

The percentage of taxonomic responses are shown in Figure 1. A one way ANOVA on the proportion of taxonomic responses with condition (control, local, global) as the independent variable yielded a significant effect of condition $F(2,96)=4.74, \mathrm{MSE}=.02, p=.011, \eta_{P}^{2}=.09$. Paired comparisons (Fisher's LSD) showed that there were significantly more taxonomic choices in the local condition than the global condition $(p=.006)$, and significantly more taxonomic choices in the control condition than the global condition $(p=.014)$. There was no difference between the control and local conditions $(p=.64)$. The findings therefore replicate that of Experiment 1 in terms of the difference in taxonomic choices between global and local conditions. However, in Experiment 1 the effect appeared to be driven by the local condition as this differed from the control condition whereas the global condition did not. In Experiment 2, the reverse was the case, with the effect being driven by the global condition and no difference between the local and control conditions. It is not clear why this is the case, and we return to this issue in the discussion of Experiment 3. 


\section{Experiment 3}

Experiments 1 and 2 both used the same method to prime processing style. Experiment 3 therefore examined whether the same findings would be replicated using a different task to prime processing style. Participants were shown black and white pictures and either wrote a caption describing the picture (global processing group) or completed a visual search task within the image for a small target (local processing group).

\section{Method}

Participants. The control, local and global groups comprised 166, 31 and 39 participants from a University in Milan, Italy.

Materials and Procedure. The experiment was the same as in Experiment 1 except for the processing style inducement task. Local processing was induced by showing participants a series of ten black and white line drawings for $15 \mathrm{~s}$ each and asking participants to search the picture for a small black and white cube and indicate where it was on an answer sheet containing the pictures but not the cubes (see Figure 2). This search task required participants to attend in detail to the objects in each picture and thus induced local processing. As in Experiments 1 and 2, to induce global processing participants were shown the same pictures and were asked to write a caption explaining what was going on in the picture. Control participants simply completed the triad task without being shown the pictures. All participants subsequently completed the triad choice task described in Experiment 1.

\section{Results and Discussion}

The percentage of taxonomic responses are shown in Figure 1. A one way ANOVA on the proportion of taxonomic responses with condition (control, local, global) as the between subjects variable yielded a significant effect of condition $F(2,236)=6.80$, MSE $=.05, p=.001, \eta_{P}^{2}=.055$. Paired comparisons (Fisher's LSD) showed that there were significantly more taxonomic choices in the local condition than the global condition $(p=.018)$, and significantly more taxonomic choices in the control 
condition than the global condition $(p<.001)$. There was no difference between the control and local conditions $(p=.68)$.

Experiment 3 again shows evidence that processing style can influence the propensity to make a taxonomic choice on the triad task, suggesting that the general findings of Experiment 1 and 2 were not dependent on the particular method used to induce processing style. Interestingly however, as in Experiment 2 the effect appeared to be driven by global processing decreasing the number of taxonomic choices. This contrasts with Experiment 1 in which local processing appeared to be increasing the number of taxonomic choices. It is unclear why these differences arise. If the difference in findings between Experiment 1 and 2 were due to the type of triad task used, then one might expect that using the same triad task as Experiment 1 in Experiment 3 would yield the same effects. This was not the case, although the different findings in Experiment 1 and 3 might have been due to the task used to induce processing. One potential issue in all these experiments is that the manipulations were made between subjects, in a classroom setting. It is plausible that this led to differences in the effectiveness of the processing inducement between groups. To address this issue, in Experiment 4 processing style was induced within subjects. In addition, participants made similarity ratings between pairs of items rather than complete a triad task. Experiment 4 therefore enabled a more controlled assessment of the effects of processing style, as well as examined whether processing style influences the sensitivity toward different taxonomic/thematic judgements and not simply the preference to make a choice based on these types of relation as measured by the triad task.

There are several other issues with Experiments 1-3 that were addressed in Experiment 4. The first was the style of inducement. In Experiments 1-3 the global processing inducement required creating a caption. There is a possibility that this was doing something in addition to inducing global processing (e.g., priming creativity). Another possibility is that the local processing inducement also had unintended effects. In Experiments 1 and 2, the change blindness task asked people to search for differences between images. In Experiment 3 a visual search task was used, and search requires an active process of comparing a representation of the target with objects in the display (e.g., Guest \& Lamberts, 2011). As there is only one target in the image, the majority of the image differs from the target representation. Thus in Experiments 1-3 it is unclear whether local processing was induced or whether the inducement simply created a focus on 
differences. As indicated in the introduction, a focus on differences might support taxonomic thinking. Thus there was the possibility that the effects observed were not caused by a global/local processing style. To that end, in Experiment 4 we used the well-established Navon task (Navon, 1977) to induce a global and local processing style.

\section{Experiment 4}

\section{Method}

Participants. Participants were 58 students from a University in Nottingham, UK and gained course credit from completing the study.

Materials and Stimuli. Processing style was induced via a Navon task (Navon, 1977) in which a large letter is briefly presented that is comprised of smaller letters. In this case the large overall letter shape could be an $\mathrm{H}$ or an $\mathrm{S}$ which was built up of smaller Hs or Ss. In the global condition participants had to respond whether the global shape was an $\mathrm{H}$ or $\mathrm{S}$ as quick as possible by pressing those keys on the keyboard and in the local condition participants responded $(\mathrm{H}$ or $\mathrm{S})$ as to the shape of the smaller letters making up the overall shape. Following Navon (1977) we used congruent trials, where the smaller letters were the same as the larger letter (e.g., a large $\mathrm{H}$ made up of smaller Hs), conflict trials, where the larger letter was made up of smaller letters from the opposite response (e.g., an $\mathrm{H}$ made up of Ss) and neutral trials in which either the larger letter was made up of smaller rectangles (global condition) or the smaller letters were rectangles (local condition).

Participants were also asked to rate the similarity between items listed in Table 1 on scale from 1 (not at all similar) to 7 (very similar). Similarity rating was used in order to gain a better understanding of the effect of processing style on the sensitivity to taxonomic/thematic relations rather than the preference to base choices on these different relations as measured by the triad task. There were two lists of stimuli used in two rating tasks, one presented after each processing style inducement (see Table 1). Within each task participants were asked to rate the similarity between the base item and the taxonomic item and the similarity of the base item to the thematic item. Questions were presented in a random order.

The Experiment was coded in Inquisit 4 (millisecond.com) and hosted on the web with Inquisit Web. 
Design and Procedure. Participants completed both the global and local task and completed a rating task after each processing style task. The order in which the global/local tasks and the rating tasks completed was counterbalanced yielding four potential orders (G-R1-L-R2, G-R2-L-R1, L-R1-G-R2, L-R2-G-R1, where $\mathrm{G}$ and $\mathrm{L}$ refer to the global and local conditions and $\mathrm{R} 1$ and $\mathrm{R} 2$ the rating task 1 or 2 ).

Counterbalancing group was automatically allocated. However, due to the online nature of the study there were many instances of participants following the online link to the study but failing to start or complete it. As such, the numbers for the counterbalancing groups were 9,17, 13, 19 (relative to the groups listed above). Due to the unequal spread of participants across counterbalancing group, this was included as a factor in the analysis.

On a given session the experiment proceeded as follows. Participants first completed a Navon task in which they were told to focus and respond relative to the global shape (global task) or the local shapes (local task). On a given trial a beep (50ms) followed by a central fixation cross $(500 \mathrm{~ms})$ signalled the start of a trial. A stimulus then appeared in one of the four quadrants of the display. In the global task the stimulus was presented for $380 \mathrm{~ms}$ and in the local task $650 \mathrm{~ms}$ (prior testing indicated longer stimulus exposure was required in the local task) after which a black and white checkerboard type mask was presented at the stimulus location which stayed onscreen until response. The inter trial interval was $400 \mathrm{~ms}$. Participants completed an 8 trial practice task followed by 24 trials of each sub-condition (congruent, conflict, neutral), randomly intermixed.

Participants then completed one of the rating tasks, which comprised 14 questions, half with thematically linked items and half with taxonomically linked items. They then completed the other processing task followed by the final rating task.

\section{Results and Discussion}

To check the efficacy of the processing style manipulation mean RTs from the Navon task were examined. First, data were trimmed by removing trials in which RTs were slower than 1000ms, leading to removal of $5.2 \%$ of trials. Mean RTs for the different sub-conditions are shown in Table 4. As is evident, RTs were generally longer for the local task and increased for conflict trials relative to consistent trials, with 
this being more evident in the local processing task. A 3 (conflict, consistent, neutral) x 2 (global, local) within subjects ANOVA therefore yielded a significant interaction $F(2,114)=19.14$, MSE $=835.75, p$ $<.001, \eta_{P}^{2}=.26$ as well as significant main effects of task $F(1,57)=208.99$, MSE $=8339.36, p$ $<.001, \eta_{P}^{2}=.79$, and sub-condition $F(2,114)=42.53, \mathrm{MSE}=838.42, p<.001, \eta_{P}^{2}=.43$. Planned post-hoc comparisons (Fisher's LSD) between the conflict and consistent sub-conditions revealed that conflict trials were significantly slower in the Local task $(p<.001)$ and the Global task $(p=.005)$. Importantly, the direction of the main effects and interactions is consistent with that found by Navon (1977). Thus, whilst not important in terms of the major research question, the Navon task data do indicate that participants completing the task online were behaving like typical participants on this task.

Mean similarity ratings are shown for the rating task in Table 5. As can be seen, similarity ratings for taxonomically related items appeared to be greater when participants had been induced with local processing compared to when induced with global processing, with the reverse true for thematically related items. A $2 \times 2 \times 4$ repeated measures ANOVA with processing style (global, local), relation (taxonomic, thematic) and counterbalancing condition (four orders) was performed on the mean similarity ratings (note that whether or not counterbalancing was included in the analysis had no effect on whether the effects reported were significant). This yielded a significant main effect of relation $F(1,54)=4.52, \mathrm{MSE}=1.32, p$ $=.038, \eta_{P}^{2}=.08$, with similarity ratings typically higher for taxonomically related items, and a significant interaction between processing style and relation, $F(1,54)=9.18, \mathrm{MSE}=.33, p=.004, \eta_{P}^{2}=.15$. Post-hoc comparisons (Fisher LSD) revealed that for taxonomic items, similarity ratings were greater when processing style was local as oppose to global $(p=.001)$ and that when processing style was local, similarity ratings were significantly higher for the taxonomic items than the thematic items $(p=.002)$. No other comparisons were significant (all $p>.48$ ).

The results of Experiment 4 replicate the general finding of an effect of processing style on preference toward taxonomic/thematic relations in the triad choice task. Importantly they extend this by showing that processing style influences the sensitivity to these different similarity relations. Specifically, In Experiment 4 this effect was driven by the local processing increasing sensitivity to taxonomic relations, replicating the general finding in Experiment 1. Importantly, this effect was shown in a within subjects 
design, ruling out the effects of any between subject differences and using a manipulation that specifically manipulated global and local processing style in a well-established way.

\section{General Discussion}

Taxonomic and thematic relations activate distinct cortical regions (e.g., Schwartz et al., 2011) and exert distinct behavioral effects (Estes et al., 2011). Assessing taxonomic relations requires examination of the ways in which features of two objects differ or are similar. Assessing thematic relations requires assessing how objects could functionally interact with each other. Thus taxonomic and thematic processing appear distinguishable in terms of the degree of abstract (thematic) versus concrete (taxonomic) thinking required and relatedly in the focus on whole objects and the relation between them (a global focus) or the parts or features of objects (a local focus) (Burgoon, Henderson \& Markman, 2013). In four experiments we therefore examined whether inducing a local/global processing style would result in taxonomic/thematic thinking. Local processing was induced via a change blindness task (Experiments 1 and 2), a visual search task (Experiment 3) or a Navon task (Experiment 4). Global processing was induced by participants creating a caption for a picture (Experiments 1-3) or a Navon task (Experiment 4).

Experiment 1 measured preference for taxonomic/thematic relations using a triad task whereby participants reported which of two items (a thematically or a taxonomically related item) was more similar to a base item. Inducing processing style influenced this preference, with more taxonomic options chosen when participants were induced with local processing. Experiment 1 also examined whether global/local processing influenced a difference/similarity focus. A difference/similarity focus is related to concrete/abstract thinking respectively (Burgoon, Henderson \& Markman, 2013) and also appears to underlie taxonomic/thematic thinking (Gentner \& Gunn, 2001; Golonka \& Estes, 2009). Inducing local/global processing did influence the number of differences/similarities listed between items although this appeared to be due to local processing increasing the number of differences listed relative to similarities. Thus there is some evidence that inducing processing style influences a focus on differences or similarities ${ }^{1}$, and this may support (or be supported by) a change in preference for taxonomic/thematic relations. 
Experiment 2 used the same method to induce processing style but used a different form of the triad task. Participants were told to imagine that they had just bought a particular product, and then they were asked which of two other products (a thematically or a taxonomically related product) they would prefer to buy. Participants induced with local processing chose more taxonomically related products than those induced with global processing. Experiment 3 showed that the effect was not dependent on the method used to manipulate processing style, as the same general effect was used when a visual search ask was used to induce local processing.

Experiment 4 addressed a number of limitations of Experiments 1-3, namely that the way in which processing style was manipulated could have had other effects besides that of attentional focus and that in Experiments 1-3 it was unclear whether local or global processing was driving the effect of processing style, potentially due to the between subjects procedure used. Experiment 4 therefore used a well-established method of inducing local/global attention, the Navon task (Navon, 1977), and manipulated processing style within subjects. Again, processing style was shown to influence sensitivity toward taxonomic/thematic relations, and this effect appeared to be driven by local processing increasing sensitivity toward taxonomic relations.

Overall, Experiments 1-4, show consistent evidence for an effect of processing style on preference for and sensitivity to taxonomic/thematic relations. What is not clear is whether this is due to local processing increasing preference for taxonomic relations (Experiments 1 and 4) or global processing increasing preference for thematic relations (Experiments 2 and 3). Theoretically, local processing should increase sensitivity toward taxonomic relations as it requires more concrete thinking and a comparison of features of objects. In contrast, this is not required for thematic processing, which requires more abstract consideration of potential ways in which two objects could functionally interact. As this requires attention to the whole object as well as more abstract thinking this may be primed by a global focus. Clearly Experiments 1-4 show that both local and global processing can play a role in terms of influencing preference for taxonomic/thematic relations, although we did not show evidence for both having effects within a single experiment. Throughout these experiments there were numerous differences in terms of the task used to induce processing style and the task used to measure sensitivity toward taxonomic/thematic 
relations. It is probable that these differences, alongside other factors might influence when global or local processing has a greater effect on the sensitivity toward taxonomic relations, and this remains an avenue for future research to explore.

The present research makes several contributions. Primarily these results show a link between global/local processing and thematic/taxonomic thinking. In previous studies preference for taxonomic/thematic relations has been shown to be stable across tasks (Mirman \& Graziano, 2012b; Simmons \& Estes, 2008). For example, Simmons and Estes (2008) showed that some participants (normally around two thirds) showed a consistent preference for either taxonomic or thematic relations. These findings suggest that the way in which people think about the relations between concepts may be relatively fixed. In contrast, the present study shows that inducing processing style can influence preference for taxonomic/thematic relations. Thus it appears that, although people may have a bias toward preferring thematic or taxonomic relations, this preference is malleable. An interesting question is the extent to which these preferences are malleable, and whether this differs depending on the initial strength of bias toward taxonomic/thematic processing.

Prior research has shown that taxonomic processing and thematic processing differ in the extent to which they rely on information about differences and commonalities (Gentner \& Gunn, 2001; Golonka \& Estes, 2009; Wisniewski \& Bassok, 1999). It is possible that a focus on differences and commonalities may support taxonomic and thematic thinking, however to date the above studies have only shown that processing mode (taxonomic or thematic) affected a focus on differences or commonalities. Here we show some limited evidence that global and local processing can also influence a focus on differences and commonalities, whereby inducing local processing increased the number of differences listed relative to commonalities. As the same experiment also showed an effect of local processing on preference for taxonomic items, this provides further support for the notion that a difference focus is related to taxonomic thinking. By showing that taxonomic and thematic processing relate to different processes, these results thus provide new and important support for dual process models of similarity and categorization (Estes, 2003; Estes et al., 2011; Wisniewski \& Bassok, 1999). 
In addition to advancing theoretical understanding, these findings also have applied implications. In Experiment 2, the method used to assess the extent to which choices were based on taxonomic/thematic relations was a triad task in which participants imagined they had just bought a particular product and were asked which of two other products (a thematically or a taxonomically related product) they would prefer to buy. Not only did inducing processing style influence the extent to which participants preferred taxonomic/thematic similarity relations, but also consumers' product choices. This highlights the importance of considering different types of product relations in consumer behaviour. For example, traditional models of product choice assume that the set of products considered in a decision are those within the same taxonomic product category (Ratneshwar, Pechmann, \& Shocker, 1996). However, it has become increasingly clear that consumers often form consideration sets that consist of items from different taxonomic categories, for example when they have a specific goal (Ratneshwar et al., 1996). In terms of thematic relations, research has shown that consumers are sensitive to thematic relations between products even when these relations are not made explicit (Felcher, Malaviya, \& McGill, 2001) and that such thematic product relations need to be positioned differently from taxonomic product relations (Noseworthy, Finlay, \& Islam, 2010). Recently, Estes, Gibbert, Guest, and Mazursky (2012) have also shown that thematic relations between a core product of a brand and a proposed brand extension have a positive impact on brand extension evaluation. The current research adds to this stream of research by demonstrating how taxonomic/thematic choices can be facilitated. 


\section{References}

Burgoon, E. M., Henderson, M. D., \& Markman, a. B. (2013). There Are Many Ways to See the Forest for the Trees: A Tour Guide for Abstraction. Perspectives on Psychological Science, 8, 501-520. http://doi.org/10.1177/1745691613497964

Derryberry, D., \& Tucker, D. M. (1994). Motivating the focus of attention. In P. M.

Niedenthal \& S. Kitayama (Eds.), Heart's eye: Emotional influences in perception and attention (pp. 167-196). New York: Academic Press.

Dijkstra, K. A., van der Pligt, J., van Kleef, G. A., \& Kerstholt, J. H. (2012). Deliberation versus intuition:

Global versus local processing in judgment and choice. Journal of Experimental Social Psychology, 48, 1156-1161

Estes, Z. (2003). A tale of two similarities: comparison and integration in conceptual combination. Cognitive Science, 27, 911-921.

Estes, Z., Gibbert, M., Guest, D., \& Mazursky, D. (2012). A dual-process model of brand extension: Taxonomic feature-based and thematic relation-based similarity independently drive brand extension evaluation. Journal of Consumer Psychology, 22, 86-101.

Estes, Z., Golonka, S., \& Jones, L. L. (2011). Thematic Thinking: The Apprehension and Consequences of Thematic Relations. Psychology of Learning and Motivation: Advances in Research and Theory, Vol 54, 249-294.

Estes, Z., Jones, L. L., \& Golonka, S. (2012). Emotion affects similarity via social projection. Social Cognition, 30, 582-607.

Felcher, E. M., Malaviya, P., \& McGill, A. L. (2001). The role of taxonomic and goalderived product categorization in, within, and across category judgments. Psychology \& Marketing, $18,865-887$.

Förster, J., \& Dannenberg, L. (2010). GLOMOsys: A systems account of global versus local processing. Psychological Inquiry, 21, 175-197.

Fujita, K., \& Roberts, J. (2010). Promoting prospective self-control through abstraction. Journal of 
Experimental Social Psychology, 46, 1049-1054.

Gentner, D. \& Brem, S. (1999). Is snow really like a shovel? Distinguishing similarity from thematic relatedness. Proceedings of the Twenty-first Annual Conference of the Cognitive Science Society, 179-184.

Gentner, D., \& Gunn, V. (2001). Structural alignment facilitates the noticing of differences. Memory \& Cognition, 9, 565-577.

Gentner, D., \& Markman, A. B. (1997). Structure mapping in analogy and similarity. American Psychologist, 52, 45-56.

Golonka, S., \& Estes, Z. (2009). Thematic relations affect similarity via commonalities. Journal of Experimental Psychology: Learning, Memory, and Cognition, 35, 1454-1464.

Guest, D., \& Lamberts, K. (2011). The time course of similarity effects in visual search. Journal of Experimental Psychology-Human Perception and Performance, 37, 1667-1688.

Howell, D., C. (2016) Fundamental Statistics for the Behavioural Sciences 9ninth edition). Cenage Learning. Boston.

Huntsinger, J. R., Clore, G. L., \& Bar-Anan, Y. (2010). Mood and global-local focus: Priming a local focus reverses the link between mood and global-local processing. Emotion, 10, 722-726.

Kalénine, S., Peyrin, C., Pichat, C., Segebarth, C., Bonthoux, F., \& Baciu, M. (2009). The sensory-motor specificity of taxonomic and thematic conceptual relations: A behavioral and fMRI study. Neuroimage, 44, 1152-1162.

Maguire, M. J., Brier, M. R., \& Ferree, T. C. (2010). EEG theta and alpha responses reveal qualitative differences in processing taxonomic versus thematic semantic relationships. Brain \& Language, 114, $16-25$.

Markman, A. B., \& Loewenstein, J. (2010). Structural comparison and consumer choice. Journal of Consumer Psychology, 20, 126-137.

Markman, A. B., \& Wisniewski, E. J. (1997). Similar and different: The differentiation of basic-level categories. Journal of Experimental Psychology: Learning, Memory, and Cognition, 23, 54-70. 
McCrea, S. M., Wieber, F., \& Myers, A. L. (2012). Construal level mind-sets moderate self- and social stereotyping. Journal of Personality and Social Psychology, 102, 51-68.

Mirman, D., \& Graziano, K. M. (2012)a. Damage to temporo-parietal cortex decreases incidental activation of thematic relations during spoken word comprehension. Neuropsychologia, 50, 1990-1997.

Mirman, D., \& Graziano, K. M. (2012)b. Individual differences in the strength of taxonomic versus thematic relations. Journal of Experimental Psychology: General, 141, 601-9.

Mullen, B., Pizzuto, C., \& Foels, R. (2002). Altering intergroup perceptions by altering prevailing mode of cognitive representation: “They look like people.” Journal of Personality and Social Psychology, 83, $1333-1343$.

Navon, D. (1977). Forest before trees: The precedence of global features in visual perception. Cognitive Psychology, 9, 353-383.

Noseworthy, T. J., Finlay, K. F., \& Islam, T. (2010). From a commodity to an experience: The moderating role of thematic positioning on congruity-based product judgment. Psychology \& Marketing, 27, 465-486.

Ratneshwar, S., Pechmann, C., Shocker, A. D. (1996). Goal-derived categories and the antecedents of across-category consideration. Journal of Consumer Research, 23, 240-250.

Schwartz, M. F., Kimberg, D. Y., Walker, G. M., Brecher, A., Faseyitan, O. K., Dell, G. S., et al. (2011). Neuroanatomical dissociation for taxonomic and thematic knowledge in the human brain. Proceedings of the National Academy of Sciences of the United States of America, 108, 8520-8524.

Simmons, S., \& Estes, Z. (2008). Individual differences in the perception of similarity and difference. Cognition, 108, 781-795.

Tversky, A. (1977). Features of similarity. Psychological Review, 84, 327-352.

Wisniewski, E. J., \& Bassok, M. (1999). What makes a man similar to a tie? Stimulus compatibility with comparison and integration. Cognitive Psychology, 39, 208-238. 


\section{Footnotes}

${ }^{1}$ Note that it has previously been suggested that inducing global or local processing increases the amount of commonalities or differences reported when comparing items, but this paper has since been retracted.

${ }^{2}$ Fisher's LSD corrections are appropriate when three or fewer post-hoc comparisons are made and will not lead to an increase in the probability of a Type 1 error (see Howell, 2016 p424-428).. When four comparisons are made, the chance of a Type 1 error increases to .1. As such, when reporting Fisher's LSD when there were four potential comparisons we corrected the $p$ values by multiplying by them by two. This correction maintains the chance of a Type 1 error at .05 . 


\section{Tables}

Table 1. Stimuli used Experiments 1, 3 (triad tasks) and 4 (similarity rating). The symbols $*$ and ${ }^{\wedge}$ denote the items used in the two rating tasks in Experiment 4

\begin{tabular}{|c|c|c|c|}
\hline Experiment & Base item & Taxonomic item & Thematic item \\
\hline & Lamp* & Flashlight & Desk \\
\hline & Sapphire $^{\wedge}$ & Emerald & Ring \\
\hline & Tent $^{\wedge}$ & Hut & Camp \\
\hline Experiments & Ship $^{\wedge}$ & Yacht & Sailor \\
\hline \multirow[t]{6}{*}{1,2 and 4} & $\operatorname{Dog} \wedge$ & Cat & Bone \\
\hline & $\mathrm{Bee}^{\wedge}$ & Butterfly & Honey \\
\hline & Squirrel$^{\wedge}$ & Rat & Nut \\
\hline & $\mathrm{Net}^{\wedge}$ & Rope & Fish \\
\hline & Fur* & Hair & Coat \\
\hline & Needle* & Pin & Thimble \\
\hline Additional & Movie* & Documentary & Producer \\
\hline Experiment & Cake* & Cookie & Birthday \\
\hline \multirow[t]{2}{*}{4 stimuli } & River* & Lake & Boat \\
\hline & Shirt* & Jacket & Tie \\
\hline
\end{tabular}


Table 2. Mean number (and standard deviations) of differences and commonalities listed in Experiment 1

Differences Commonalities

\begin{tabular}{ccc}
\hline Local Processing (Overall) & $10.58(4.18)$ & $7.17(8.75)$ \\
Global Processing (Overall) & $9.43(2.90)$ & $8.46(2.13)$ \\
Local Processing (Taxonomic) & $10.21(4.13)$ & $6.30(1.42)$ \\
Global Processing (Taxonomic) & $8.87(3.00)$ & $7.33(2.27)$ \\
Local Processing (Thematic) & $.38(.77)$ & $.87(.92)$ \\
Global Processing (Thematic) & $.57(.79)$ & $1.13(.90)$
\end{tabular}


Table 3. Stimuli used in the triad task in Experiment 2.

\begin{tabular}{ccc} 
Base item & Taxonomic item & Thematic item \\
\hline TV & Stereo & Sofa \\
Computer & Printer & Desk \\
Coffee & Juice & Newspaper \\
Running Shoes & Shorts & Ipod \\
Pot & Pan & Eggs \\
Shelves & Cupboard & Screwdriver \\
Shampoo & Conditioner & Towel \\
Flowers & Plant & Vase \\
Rain Coat & Sweater & Umbrella \\
Wine & Beer & Chocolate \\
Pen & Pencil & Notebook \\
Cake & Cookies & Candles
\end{tabular}


Table 4. Mean RTs (and standard deviations) for the Navon task in Experiment 4.

Consistent Conflict Neutral

\begin{tabular}{cccc}
\hline Local Processing & $570.89(80.59)$ & $626.99(94.66)$ & $613.20(80.44)$ \\
Global Processing & $456.60(101.58)$ & $469.27(103.68)$ & $460.60(100.06)$
\end{tabular}


Table 5. Mean similarity ratings (and standard deviations) for taxonomically and thematically related items in Experiment 4.

$\begin{array}{ll}\text { Taxonomically } & \text { Thematically } \\ \text { related items } & \text { related items }\end{array}$

Local Processing $\quad 4.60(1.10) \quad 4.06(1.41)$

Global Processing $\quad 4.26(1.07) \quad 4.14(1.48)$ 


\section{Figure Captions}

Figure 1. Mean percentage of taxonomic choices made in Experiments 1-3. Error bars indicate standard errors.

Figure 2. Stimuli used to induce processing style in Experiment 3. Note that the arrow in panel C is to aid the reader and was not shown to participants. 


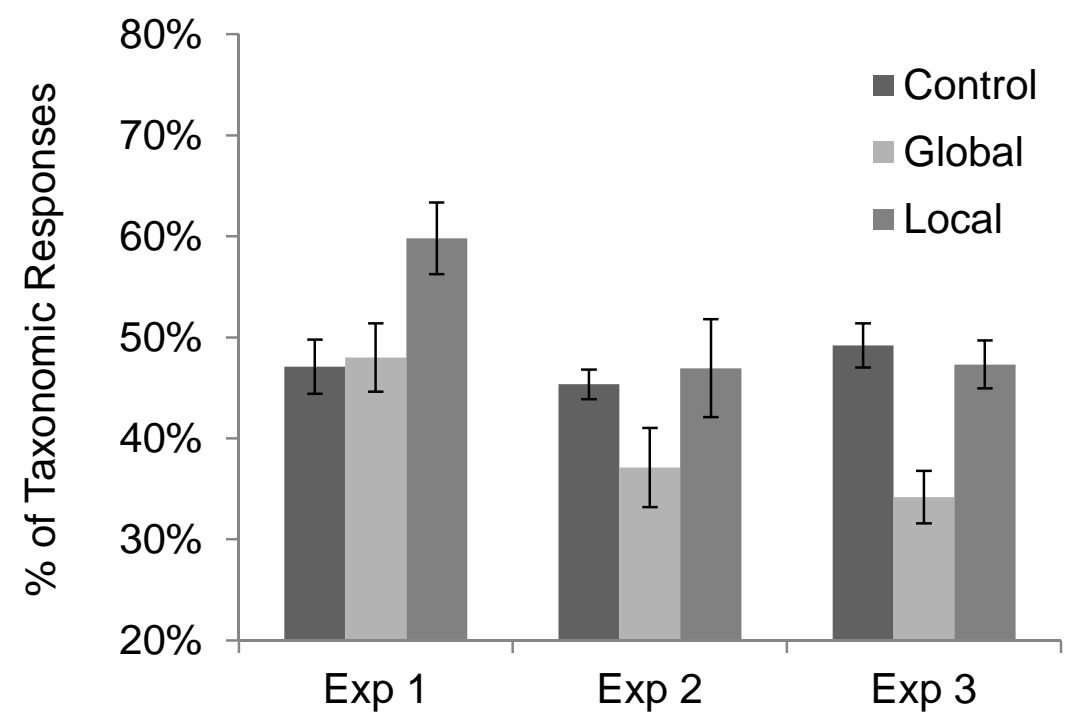

Figure 1 


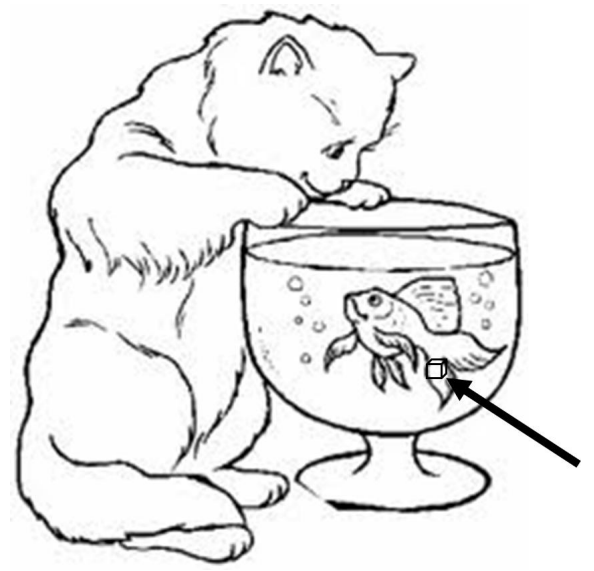

Figure 2. 